\title{
ATRIBUTOS FÍSICOS E REQUERIMENTO DE POTÊNCIA DE UMA SEMEADORA- ADUBADORA EM UM LATOSSOLO SOB ESTADOS DE COMPACTAÇÃO ${ }^{1}$
}

\author{
ANDRÉIA K. BONINI ${ }^{2}$, ANTONIO GABRIEL FILHO ${ }^{3}$, DEONIR SECCO ${ }^{3}$, \\ RENAN F. DE SOUZA ${ }^{4}$, CELSO TAVARES ${ }^{5}$
}

RESUMO: Este experimento foi desenvolvido em um Latossolo, no Núcleo Experimental de Engenharia Agrícola, localizado em Cascavel - PR, com o objetivo de avaliar os atributos físicos e o esforço tratório de semeadora-adubadora em quatro estados de compactação, e sua influência na população inicial das culturas de soja e milho. O delineamento consistiu em esquema "Split Block", com dois fatores, sendo duas culturas (soja e milho) no primeiro fator e quatro estados de compactação no segundo fator, totalizando oito tratamentos. Os tratamentos foram: T0 - sem compactação adicional; T1 - com uma passada; T3 - com três passadas, e T5 - com cinco passadas de rolo compactador. Avaliaram-se a profundidade do sulco, a velocidade média de deslocamento, a força e a potência requerida na barra de tração na operação de semeadura, a densidade e a resistência do solo à penetração e, ainda, a população inicial das culturas. Com os resultados observados, pode-se concluir que a pressão aplicada pelo disco duplo defasado não foi suficiente para manter a mesma profundidade de sulco no maior estado de compactação. A resistência à penetração do solo de 2,50 e $3,00 \mathrm{MPa}$ e a densidade do solo máxima de $1,39 \mathrm{Mg} \mathrm{m}^{-3}$ não interferiram na população inicial de plantas de soja, no entanto, para o milho, ocorreu redução nos tratamentos. À medida que ocorreu variação na profundidade de sulcamento e na velocidade de deslocamento, aumentou a força de tração por linha para as duas culturas estudadas.

PALAVRAS-CHAVE: densidade do solo, máquina agrícola, tração.

\section{PHYSICALS ATTRIBUTES AND POWER REQUIREMENT OF A PLANTER IN AN OXISOL COMPACTED SOIL}

\begin{abstract}
This experiment was conducted in an Oxisol, in the Experimental Center of Agricultural Engineering, located in Cascavel (PR), Brazil, with the objective of evaluates the physicals attributes and the tractor effort of a planter in four levels of compactation and the influence in the initial population in the cultures of soybean and corn. The experimental outliner scheme was "Split Block", tested with two factors, being two cultures (soy and corn) the first factor and the four levels of compactation the second one, totalizing eight treatments. The four levels of compaction were determined as: T0 - without compaction; T1 - with one pass of the soil compact roll; T3 - with tree, and T5 - with five passes. The parameters evaluated were: the depth of the furrow, average speed, traction force and power force, soil bulk density, soil penetration resistance and the stand of the soybean and corn cultures. The results showed that the applied pressure by the double unbalanced record was not sufficient to maintain the furrow depth in major compactation level. The soil penetration resistance 2.50 and $3.00 \mathrm{MPa}$ and the maximum bulk density $1.39 \mathrm{Mg} \mathrm{m}^{-3}$ did not influence the initial population of the soybean culture, however with the corn culture occurred a reduction in the treatments. When the variation in the depth and the speed of the movement occurred, the strength of traction per line for both cultures studied increased.
\end{abstract}

KEYWORDS: bulk density, agricultural machine, traction.

\footnotetext{
${ }^{1}$ Extraído da dissertação de mestrado do primeiro autor.

${ }^{2}$ Discente do PPGEA, Centro de Ciências Exatas e Tecnológicas, UNIOESTE, Cascavel - PR, Fone: (0XX45) 3220-3254, akbonini@yahoo.com.br

${ }^{3}$ Eng $^{\mathrm{o}}$ Agro $^{-}$, Professor Doutor, Centro de Ciências Exatas e Tecnológicas, UNIOESTE, Cascavel - PR.

${ }^{4}$ Discente do Curso de Engenharia Agrícola, Centro de Ciências Exatas e Tecnológicas, UNIOESTE, Cascavel - PR

${ }^{5}$ Discente do Programa de Pós-Graduação em Engenharia Agrícola, UNIOESTE.

Recebido pelo Conselho Editorial em: 7-8-2006
}

Aprovado pelo Conselho Editorial em: 14-1-2008 


\section{INTRODUÇÃO}

A operação de semeadura/adubação é decisiva no estabelecimento de culturas anuais para produção de grãos. Nos sistemas conservacionistas, sua importância aumenta visto que as condições de solo e de cobertura geralmente dificultam a deposição das sementes. Trabalhos realizados junto a agricultores identificaram que as semeadoras-adubadoras utilizadas no plantio direto têm apresentado problemas de desempenho em solos com altos teores de argila. A alta resistência aos componentes rompedores que ocorrem nesses solos, associada a sua grande retenção de umidade, tem proporcionado constante adaptação das máquinas, especialmente em busca de soluções para os problemas, como: corte da palha, dosagem das sementes e do adubo nas quantidades preestabelecidas, aderência do solo nos componentes, desuniformidade na profundidade de semeadura, cobertura e compactação irregular do solo sobre as sementes que acabam afetando a uniformidade e a emergência das plantas, além de exigir maior esforço tratório (SILVA \& ROSOLEN, 2002; MERCANTE et al., 2005).

No entanto, em áreas onde o sistema plantio direto já está implantado há muito tempo, podem ocorrer problemas sérios de compactação superficial, ocasionada principalmente pelo tráfego constante das máquinas ou, ainda, devido à utilização inadequada de técnicas de semeadura direta na área agrícola (SOANE \& OUWERKERK, 1994; CEPIK et al., 2005). Segundo OLIVEIRA et al. (2000), houve, nesses últimos anos, grande evolução nas semeadoras-adubadoras, principalmente no mecanismo de distribuição de sementes. Dessa forma, nas semeadoras, há várias combinações e arranjos de mecanismos dosadores de sementes, de fertilizantes e de elementos rompedores de solo que conferem a elas diferentes exigências de força para serem tracionadas.

CEPIK et al. (2005) estudaram a força de tração em um Argissolo sob plantio direto e concluíram que houve aumento na demanda de tração com o aumento da profundidade trabalhada, e sua magnitude dependeu do estado de consistência do solo, sendo verificado, também, que a velocidade não influenciou na força de tração.

FURLANI et al. (2005) encontraram resultados semelhantes com relação à velocidade em Latossolo Vermelho. Entretanto, para a força de tração, verificaram que não ocorreu diferença entre os preparos do solo. Em estudo com semeadura de aveia, SILVEIRA et al. (2005) verificaram que, com o aumento da profundidade de deposição de 1,97 para $2,68 \mathrm{~cm}$, o requerimento de força de tração cresceu de 3,78 para 5,51, e o aumento da velocidade de deslocamento, de 5,24 para $7,09 \mathrm{~km} \mathrm{~h}^{-1}$, acrescentou $6,9 \%$ no requerimento de força de tração.

SIQUEIRA et al. (2001) avaliaram quatro semeadoras-adubadoras em sistema plantio direto de soja. Verificaram aumento significativo no requerimento de força de tração e na potência na barra de tração quando a velocidade aumentou de 4,7 para $8,3 \mathrm{~km} \mathrm{~h}^{-1}$. Em áreas de plantio direto com 12 anos, SILVA et al. (2000) constataram que, na semeadura direta do milho (quatro linhas), os maiores valores de força de tração $(16,12 \mathrm{kN})$ ocorreram na maior velocidade.

MAHL et al. (2004), em Nitossolo Vermelho, estudaram velocidades de deslocamento e sistemas de manejos do solo e relataram que a velocidade não foi influenciada pelo sistema de manejo, entretanto, em relação às velocidades, nota-se que, na maior velocidade $\left(8,1 \mathrm{~km} \mathrm{~h}^{-1}\right)$, há maior força de tração e incremento na potência da barra de $96 \%$. Por outro lado, TRINTIN et al. (2005) não observaram o efeito da velocidade para os parâmetros forças de tração média e máxima; entretanto, verificaram o aumento do consumo horário de combustível e potências média e máxima.

Estudos realizados em Latossolo Vermelho distrófico por BORTOLLOTO et al. (2005) mostraram que tanto a força de tração quanto as demandas de potências média e máxima foram influenciadas pelo tipo de cobertura de solo e pela velocidade de deslocamento. MERCANTE et al. (2005), ao estudarem duas semeadoras, notaram que a velocidade de deslocamento quando passa de 5,20 para $8,45 \mathrm{~km} \mathrm{~h}^{-1}$, somente a potência na barra aumenta significativamente.

Em vista do exposto, este trabalho teve como objetivo determinar o requerimento de força e de potência na barra de tração de uma semeadora-adubadora na semeadura da soja e do milho sob quatro estados de compactação, verificando suas relações com a população inicial de plantas. 


\section{MATERIAL E MÉTODOS}

O experimento foi conduzido no Núcleo Experimental de Engenharia Agrícola da UNIOESTE, situado geograficamente nas coordenadas $24^{\circ} 48^{\prime}$ de latitude sul e $53^{\circ} 26^{\prime}$ de longitude oeste, altitude média de $760 \mathrm{~m}$ e declividade menor que $0,03 \mathrm{~m} \mathrm{~m}^{-1}$ na área estudada. Segundo Köeppen, o clima é classificado como cfc. Segundo a EMBRAPA (1999a), o solo da região é classificado como Latossolo Vermelho distroférrico típico, substrato basalto e relevo suave ondulado. A área destinada à implantação do projeto foi cultivada há oito anos em sistema de plantio direto, predominantemente com as culturas de aveia ou trigo, no inverno, e de milho ou soja, no verão.

O experimento foi dividido em duas fases experimentais: primeira fase - aplicação dos tratamentos com estados de compactação, utilizando rolo compactador de Marca Cartepillar, modelo CP 533D, com superfície lisa e dispositivo vibratório com 16,6 N. Foram, então, estabelecidos os seguintes estados de compactação: uma passada com rolo compactador - T1; três passadas com rolo compactador - T3; cinco passadas com rolo compactador - T5, e a unidade experimental sem compactação adicional - T0; segunda fase - implantação das culturas de soja e de milho. O delineamento experimental consistiu em experimento em faixas ("Split Block"), com dois fatores em estudo: duas culturas (soja e milho) e quatro estados de compactação, totalizando oito tratamentos, com quatro repetições, aleatorizados nas faixas de 10 x $50 \mathrm{~m}$; no momento da implantação do experimento, a umidade média do solo em cada tratamento foi: T1 $\left(0,37 \mathrm{~kg} \mathrm{~kg}^{-1}\right)$; T3 $\left(0,36 \mathrm{~kg} \mathrm{~kg}^{-1}\right)$; T5 $\left(0,38 \mathrm{~kg} \mathrm{~kg}^{-1}\right)$, e T0 $\left(0,37 \mathrm{~kg} \mathrm{~kg}^{-1}\right)$.

Antes e após a aplicação do experimento, foi realizada a caracterização de atributos físicos do solo, seguindo a metodologia da EMBRAPA (1999b), na qual foram analisados: a densidade do solo (Ds), densidade de partículas (Dp) e macroporosidade (Ma). Amostras de solo parcialmente deformadas foram coletadas na profundidade de 0 a $0,30 \mathrm{~m}$, no início do estudo, sendo secas ao ar e submetidas à análise de densidade de partículas, pelo método do balão volumétrico. A densidade do solo foi avaliada por meio da coleta de amostras indeformadas de solo, pelo método do anel volumétrico, entre as profundidades de 0 a 0,10 e 0,10 a 0,20 m. Em cada unidade experimental, foram coletadas amostras de solo em cinco pontos aleatórios, totalizando 30 amostras, com duas repetições em cada profundidade. $\mathrm{Na}$ Tabela 1, estão apresentados os dados relativos à caracterização de atributos físicos do solo antes da implantação dos tratamentos.

TABELA 1. Caracterização de atributos físicos em cada unidade experimental antes da aplicação dos tratamentos, nas profundidades de 0 a 0,10 e 0,10 a $0,20 \mathrm{~m}$. Characterization of physical attributes in each experimental unit before the implementation of the treatments at depths from 0 a 0,10 and 0,10 a $0,20 \mathrm{~m}$.

\begin{tabular}{lcc}
\hline Atributo Físico & \multicolumn{2}{c}{ Profundidade $(\mathrm{m})$} \\
\cline { 2 - 3 } & 0 a 0,10 & 0,10 a 0,20 \\
\hline Densidade do solo $\left(\mathrm{Mg} \mathrm{m}^{-3}\right)$ & 1,22 & 1,22 \\
Macroporos $\left(\mathrm{dm}^{3} \mathrm{dm}^{-3}\right)$ & 0,10 & 0,10 \\
Densidade de partículas $\left(\mathrm{Mg} \mathrm{m}^{-3}\right)$ & 2,77 & 2,77 \\
\hline
\end{tabular}

A resistência à penetração do solo foi mensurada até a profundidade de $0,45 \mathrm{~m}$, após a aplicação dos tratamentos com estados de compactação, utilizando penetrômetro eletrônico, desenvolvido por TIEPPO (2004), com ângulo do cone de $30^{\circ}$ e diâmetro da base de $12,83 \mathrm{~mm}$ (ASAE, 1999). Para cada tratamento, foram realizadas dez medições, sendo as médias por camada ( 0 a 0,1 e 0,10 a $0,20 \mathrm{~m})$ submetidas à análise estatística. A umidade gravimétrica foi determinada utilizando o método da estufa.

Para a implantação das culturas, utilizou-se de semeadora-adubadora de precisão, modelo SMT 6414, de arrasto, com mecanismo sulcador tipo facão, para deposição de adubo, e duplo disco defasado para sementes. A semeadora-adubadora foi tracionada na quarta marcha, com transmissão reduzida, rotação de $2.000 \mathrm{rpm}$, por trator marca FORD, modelo 7630 4X2, com tração dianteira auxiliar, com 75,8 kW (103 cv) de potência no motor. A semeadora-adubadora foi configurada com cinco fileiras para efetuar a semeadura da soja e regulada com espaçamento entre linhas de $0,45 \mathrm{~m}$ e 
profundidade de semeadura de $10 \mathrm{~cm}$. Para a cultura do milho, foi adotada a configuração de três fileiras, com espaçamento entre fileiras de $0,9 \mathrm{~m}$ e profundidade de semeadura de $10 \mathrm{~cm}$. A cultivar de soja utilizada foi a COODETEC 216 (CD 216), sendo semeadas 20 sementes por metro. Para o milho, foi utilizado a cultivar COODETEC 206 (CD 206) precoce, sendo semeadas seis sementes por metro.

O requerimento de força de tração na semeadora foi medido por meio de célula de carga, com capacidade para $50 \mathrm{kN}$, inserida entre a barra de tração do trator e o cabeçalho da semeadora. As forças de tração instantânea foram armazenadas no "Datalogger" e, por meio de programas computacionais, foi determinado o valor da força média dos dados armazenados para cada unidade experimental. A força de tração e a demanda de potência por linha foram determinadas na divisão pelo número de fileiras, 5 e 3 para as culturas de soja e de milho, respectivamente. A velocidade de deslocamento foi determinada medindo-se, com um cronômetro, o tempo para percorrer os $50 \mathrm{~m}$ de cada parcela. Para avaliar a profundidade do sulco, retirou-se solo mobilizado, em cada ponto de amostragem, e mensurou-se por meio de régua graduada a profundidade de mobilização do solo nos quatro tratamentos. Em cada tratamento, foram amostradas dez repetições.

A população inicial das culturas de soja e de milho foi determinada 18 dias após semeadura, logo após a estabilização da emergência. Para a contagem do número de plantas, demarcou-se aleatoriamente $1 \mathrm{~m}$ na linha de semeadura, com dez repetições cada tratamento, e realizou-se a contagem de plantas emergidas por metro.

As principais variáveis estudadas foram: profundidade do sulco de semeadura, força de tração e potência requerida na barra e, secundariamente, foram avaliadas a densidade do solo e a resistência do solo à penetração nos quatro estados de compactação. Os resultados obtidos no requerimento de potência foram submetidos à análise de variância, e as médias, comparadas entre si, pelo teste de Tukey, a 5\% de significância. Para a comparação de médias da densidade do solo e da resistência do solo à penetração, foi realizado o teste t-Student, a 5\% de significância.

\section{RESULTADOS E DISCUSSÃO}

Os resultados das análises de densidade do solo (Ds) e do volume de macroporos, nas profundidades de 0 a 0,10 e 0,10 a $0,20 \mathrm{~m}$, estão apresentados na Tabela 2. Observa-se que, nas profundidades de 0 a 0,10 e 0,10 a $0,20 \mathrm{~m}$, os valores médios de Ds obtiveram incremento com o aumento do número de passadas do rolo compactador, visto que os tratamentos T1, T3 e T5 diferiram do tratamento $\mathrm{T} 0$. $\mathrm{Na}$ avaliação desse atributo, verifica-se que os maiores valores para o tratamento T5 foram encontrados na profundidade de 0,10 a $0,20 \mathrm{~m}$, e para o tratamento T3, foram observados na profundidade de 0 a $0,10 \mathrm{~m}$. Com relação ao volume de macroporos, verifica-se comportamento inverso ao da Ds, ou seja, com o incremento da Ds, ocorreu decréscimo significativo do volume de macroporos em relação ao tratamento T0, em ambas as profundidades.

SECCO et al. (2004) também verificaram em um Latossolo que os maiores valores de Ds ocorreram nos tratamentos com três e cinco passadas com rolo compactador, na profundidade de 0 a 0,10 m. Entretanto, segundo REINERT et al. (2001), esses valores apresentados não ultrapassam a 1,45 $\mathrm{Mg} \mathrm{m}^{-3}$; sendo assim, o solo não é considerado compactado. SILVEIRA et al. (2005) observaram valores médios de $1,31 \mathrm{Mg} \mathrm{m}^{-3}$ antes da operação com a semeadura; dessa forma, observa-se valor semelhante de Ds para os tratamentos com maior estado de compactação. TRINTIN et al. (2005) relataram Ds na faixa de 1,49 a $1,51 \mathrm{Mg} \mathrm{m}^{-3}$ e ainda MERCANTE et al. (2005) encontraram valor na faixa de 1,16 a $1,28 \mathrm{Mg} \mathrm{m}^{-3}$, na profundidade de 0 a $0,15 \mathrm{~m}$, e na profundidade 0,10 a $0,30 \mathrm{~m}$, os valores de 1,17 a $1,22 \mathrm{Mg} \mathrm{m}^{-3}$.

De acordo com a Tabela 3 , observa-se que o valor médio de resistência à penetração $(\mathrm{Rp})$ do tratamento T0 diferiu do tratamento $\mathrm{T} 5$, nas duas profundidades analisadas, sendo verificado o menor valor de $\mathrm{Rp}$ no tratamento $\mathrm{TO}$, devido à não-ocorrência de tráfego pelo rolo compactador. Nota-se, ainda, que somente na profundidade de 0,10 a $0,20 \mathrm{~m}$ os tratamentos diferem entre si. Pode-se verificar que, nessa camada, em geral, encontram-se os maiores valores de Rp, principalmente nos tratamentos T3 e T5, nos quais são observados valores de 2,02 e 2,50 MPa, respectivamente. 
TABELA 2. Densidade do solo - Ds $\left(\mathrm{Mg} \mathrm{m}^{-3}\right)^{-}$e macroporosidade - $\mathrm{Ma}\left(\mathrm{dm}^{3} \mathrm{dm}^{-3}\right)$ - em função dos quatro estados de compactação. Soil Density - Ds $\left(\mathbf{M g ~ m}^{-3}\right)$ - and macroporosity Ma $\left(\mathrm{dm}^{3} \mathrm{dm}^{-3}\right)$ - according to the four compaction states.

\begin{tabular}{ccccc}
\hline \multirow{3}{*}{ Tratamentos } & \multicolumn{4}{c}{ Profundidade (m) } \\
\cline { 2 - 5 } & Ds & Ma 0,10 & Ds & Ma $10 \mathrm{a} 0,20$ \\
\cline { 2 - 5 } & $1,23 \mathrm{~b}^{*}$ & $0,11 \mathrm{a}^{*}$ & $1,23 \mathrm{c}^{*}$ & $0,10 \mathrm{a}$ \\
T0 & $1,31 \mathrm{a}$ & $0,04 \mathrm{~b}$ & $1,27 \mathrm{~b}$ & $0,04 \mathrm{~b}$ \\
T1 & $1,37 \mathrm{a}$ & $0,03 \mathrm{~b}$ & $1,34 \mathrm{a}$ & $0,02 \mathrm{c}$ \\
T3 & $1,35 \mathrm{a}$ & $0,03 \mathrm{~b}$ & $1,39 \mathrm{a}$ & $0,02 \mathrm{c}$ \\
T5 & 5,34 & 45,42 & 6,18 & 41,1 \\
C.V. $(\%)$ &
\end{tabular}

*Médias seguidas de letra iguais na coluna não diferem entre si, pelo teste t-Student $(\mathrm{P}>0,05)$.

TABELA 3. Resistência do solo à penetração (MPa) em quatro estados de compactação do solo, nas profundidades de 0 a 0,10 e 0,10 a $0,20 \mathrm{~m}$. Soil resistance to penetration (MPa) in four states of soil compaction at depths from 0 a 0,10 and 0,10 a $0,20 \mathrm{~m}$.

\begin{tabular}{lccc}
\hline & \multicolumn{3}{c}{ Profundidade $(\mathrm{m})$} \\
\cline { 2 - 4 } Tratamentos & $0 \mathrm{a} 0,10$ & $0,10 \mathrm{a} 0,20$ & Média \\
\cline { 2 - 4 } & & Resistência à Penetração & 1,24 \\
T0 & $1,27 \mathrm{c} *$ & $1,42 \mathrm{~d}$ & 1,65 \\
T1 & $1,37 \mathrm{bc}$ & $1,80 \mathrm{c}$ & 1,78 \\
T3 & $1,61 \mathrm{~b}$ & $2,02 \mathrm{~b}$ & 2,03 \\
T5 & $2,04 \mathrm{a}$ & $2,50 \mathrm{a}$ & \\
C.V. $(\%)$ & 48,05 & 29,48 & .
\end{tabular}

*Médias seguidas de letra iguais na coluna não diferem entre si, pelo teste t- Student $(\mathrm{P}>0,05)$.

Na Figura 1, observa-se a resistência do solo à penetração $(\mathrm{Rp})$ e a umidade gravimétrica do solo no dia do ensaio. Os resultados demonstram aumento nos pontos de maior $\mathrm{Rp}$ com o incremento no número de passadas do rolo compactador, na camada de 0 a $0,20 \mathrm{~m}$ de profundidade. Os maiores valores de Rp foram encontrados nos tratamentos T3 e T5 com rolo compactador, com extremos de 2,50 a 3,00 $\mathrm{MPa}$, entre as profundidades de 0,07 a $0,12 \mathrm{~m}$, mostrando que ocorreu aumento da $\mathrm{Rp}$ de acordo com número de passadas do rolo compactador, na camada de 0 a $0,20 \mathrm{~m}$ de profundidade.

O maior valor de Rp observado nos tratamentos deveu-se ao efeito acumulativo de tensões ocasionadas por máquinas ou implementos que o solo recebe em uma safra ou de uma safra para outra e, ainda, pelo fato de existir a acomodação natural das partículas mais finas pelo perfil do solo, que podem diretamente influenciar no aumento da resistência. Segundo autores como SILVA (2003), GENRO JÚNIOR et al. (2004) e SECCO et al. (2004), o confinamento dos valores mais altos de Rp, em camadas ao redor de 0,07 a 0,12 $\mathrm{m}$ de profundidade, parece ser característica de solos sob sistema plantio direto, tanto de solos argilosos como de arenosos.

CEPIK et al. (2005) observaram valores de resistência do solo à penetração na camada de 0 a $0,14 \mathrm{~m}$ de 1,93 MPa. Valores semelhantes foram encontrados neste estudo, com os estados de compactação e nessa mesma profundidade.

No tratamento sem compactação adicional (T0), a resistência do solo não ultrapassou a $1,50 \mathrm{MPa}$ entre as profundidades de 0,07 a $0,12 \mathrm{~m}$. Já os valores do T1 não tiveram grande variação, ou seja, a partir da profundidade de 0,1 a $0,35 \mathrm{~m}$, a $\mathrm{Rp}$ foi uniforme (1,70 MPa).

Na Tabela 4, estão apresentados os resultados obtidos de profundidade de sulco, velocidade média, força de tração por linha e potência na barra de tração por linha de semeadura, nas culturas de soja e de milho. Observa-se que os valores de profundidade do sulco e de velocidade média não diferem entre si em relação aos estados de compactação e também em relação às culturas de soja e de milho. 


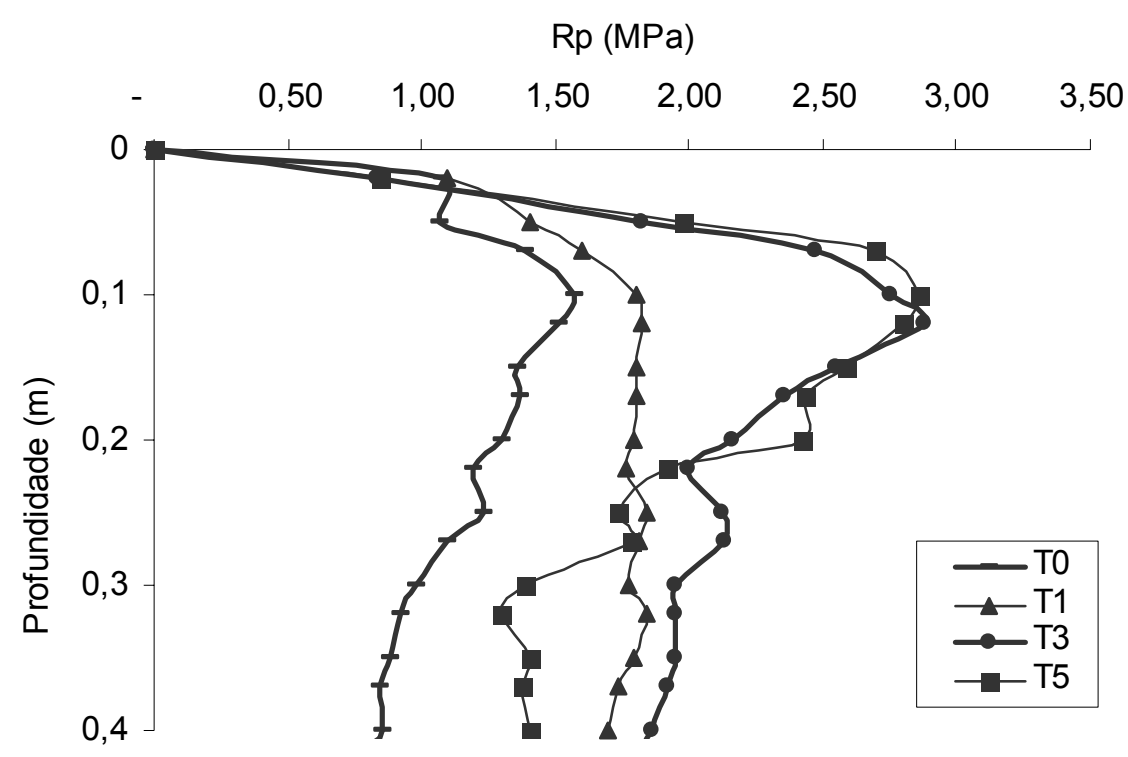

FIGURA 1. Resistência do solo em Latossolo Vermelho sob quatro estados de compactação. $\mathrm{T} 0\left(\mathrm{Ug}=0,20 \mathrm{~kg} \mathrm{~kg}^{-1}\right)$; T1 $\left(\mathrm{Ug}=0,19 \mathrm{~kg} \mathrm{~kg}^{-1}\right) ; \mathrm{T} 3\left(\mathrm{Ug}=0,18 \mathrm{~kg} \mathrm{~kg}^{-1}\right)$, e T5 $\left(\mathrm{Ug}=0,21 \mathrm{~kg} \mathrm{~kg}^{-1}\right)$. Resistance of penetration in Latosol red soil under four compaction states $\mathrm{T0}\left(\mathrm{Ug}=0,20 \mathrm{~kg} \mathrm{~kg}^{-1}\right) ; \mathrm{T} 1\left(\mathrm{Ug}=0,19 \mathrm{~kg} \mathrm{~kg}^{-1}\right) ; \mathrm{T} 3(\mathrm{Ug}=$ $\left.0,18 \mathrm{~kg} \mathrm{~kg}^{-1}\right)$, and T5 $\left(\mathrm{Ug}=0,21 \mathrm{~kg} \mathrm{~kg}^{-1}\right)$.

TABELA 4. Valores médios da profundidade de sulco (Prof), da velocidade média (Vm), da força de tração por linha $(\mathrm{Ft})$ e potência na barra de tração por linha $(\mathrm{Pb})$ de semeadura da soja (SO) e do milho (MI). Average values of the depth of furrow (Prof), the average speed $(\mathrm{Vm})$, the force of traction per line $(\mathrm{Ft})$ and power in the bar traction per line (Pb) from sowing of soybean (SO) and maize (MI).

\begin{tabular}{|c|c|c|c|c|c|c|c|c|}
\hline \multirow{2}{*}{ Fatores } & \multicolumn{2}{|c|}{ Prof. (cm) } & \multicolumn{2}{|c|}{$\operatorname{Vm}\left(\mathrm{km} \mathrm{h}^{-1}\right)$} & \multicolumn{2}{|c|}{ Ft $\left(\mathrm{kN}\right.$ linha $\left.^{-1}\right)$} & \multicolumn{2}{|c|}{$\mathrm{Pb}\left(\mathrm{kW}\right.$ linha $\left.^{-1}\right)$} \\
\hline & SO & MI & $\mathrm{SO}$ & MI & SO & MI & $\mathrm{SO}$ & $\mathrm{MI}$ \\
\hline T0 & $10,55 \mathrm{aA}$ & $11,90 \mathrm{aA}$ & $5,95 \mathrm{aA}$ & $5,90 \mathrm{aA}$ & $1,91 \mathrm{aA}$ & $1,25 \mathrm{aB}$ & $3,17 \mathrm{aA}$ & $3,41 \mathrm{aA}$ \\
\hline $\mathrm{T} 1$ & 9,90 aA & $10,75 \mathrm{aA}$ & $6,15 \mathrm{aA}$ & $6,12 \mathrm{aA}$ & $1,89 \mathrm{aA}$ & $1,40 \mathrm{aB}$ & $3,23 \mathrm{aB}$ & $3,97 \mathrm{aA}$ \\
\hline $\mathrm{T} 3$ & $10,37 \mathrm{aA}$ & $9,90 \mathrm{aA}$ & $5,90 \mathrm{aA}$ & $6,00 \mathrm{aA}$ & $1,94 \mathrm{aA}$ & $1,29 \mathrm{aB}$ & 3,18 aA & 3,58 aA \\
\hline T5 & $9,05 \mathrm{aA}$ & $9,23 \mathrm{aA}$ & $6,05 \mathrm{aA}$ & $5,85 \mathrm{aA}$ & $1,89 \mathrm{aA}$ & $1,30 \mathrm{aB}$ & 3,17 aA & 3,51 aA \\
\hline$\overline{C . V .1(\%)}$ & \multicolumn{2}{|c|}{8,04} & \multicolumn{2}{|c|}{2,09} & \multicolumn{2}{|c|}{14,89} & \multicolumn{2}{|c|}{13,34} \\
\hline C.V.2(\%) & \multicolumn{2}{|c|}{11,01} & \multicolumn{2}{|c|}{2,91} & \multicolumn{2}{|c|}{7,11} & \multicolumn{2}{|c|}{9,13} \\
\hline C.V.3(\%) & \multicolumn{2}{|c|}{4,33} & \multicolumn{2}{|c|}{1,51} & \multicolumn{2}{|c|}{4,88} & \multicolumn{2}{|c|}{5,59} \\
\hline $\mathrm{T} * \mathrm{~V}$ & \multicolumn{2}{|c|}{$0,23 \mathrm{~ns}$} & \multicolumn{2}{|c|}{$0,23 \mathrm{~ns}$} & \multicolumn{2}{|c|}{$0,87 \mathrm{~ns}$} & \multicolumn{2}{|c|}{$0,71 \mathrm{~ns}$} \\
\hline$\overline{\mathrm{DMS}(\mathrm{V})}$ & \multicolumn{2}{|c|}{3,04} & \multicolumn{2}{|c|}{0,47} & \multicolumn{2}{|c|}{0,34} & \multicolumn{2}{|c|}{0,82} \\
\hline $\mathrm{DMS}(\mathrm{T})$ & \multicolumn{2}{|c|}{2,07} & \multicolumn{2}{|c|}{0,32} & \multicolumn{2}{|c|}{0,24} & \multicolumn{2}{|c|}{0,58} \\
\hline
\end{tabular}

"Médias seguidas de mesma letra minúscula na coluna e maiúscula na linha não diferem significativamente entre si, pelo teste de Tukey, a $5 \%$ de probabilidade; $\left(\mathrm{T}^{*} \mathrm{~V}\right)$ fator estados de compactação e variedade, respectivamente, ns representa que não há interação entre os fatores em estudo ( $p>0,05)$, DMS- diferença mínima significativa e C.V. coeficiente de variação.

SILVEIRA et al. (2005) relataram resultados semelhantes para a profundidade de sulco em uma semeadora de 14 linhas, para cultura da aveia. Entretanto, com o aumento da Ds e Rp, houve tendência em diminuir a profundidade do sulco; isso pode ser verificado no tratamento T5, que teve redução de profundidade de sulco de cerca de $22,44 \%$ em relação ao tratamento T0, mostrando que, nesse estado de compactação, o sulcador não atingiu a profundidade desejada $(0,12 \mathrm{~m})$.

Nota-se que a velocidade média de deslocamento não foi afetada pela profundidade do sulco e, em geral, manteve aproximadamente a mesma entre as culturas e os estados de compactação, sendo o tratamento T1 o que obteve maior valor de velocidade média. Apesar de obter maiores valores de velocidade, essas não foram suficientes para determinar diferença significativa na força de tração e na demanda de potência por linha em relação aos demais tratamentos. 
Observa-se, ainda, que, mesmo mantendo a velocidade e a profundidade de sulco constantes para as culturas de soja e de milho, a força na barra de tração por linha diferiu significativamente para as culturas estudadas. MAHL et al. (2004) e FURLANI et al. (2005) observaram que, na maior velocidade, há maior força de tração. Por outro lado, TRINTIN et al. (2005) não observaram o efeito da velocidade para os parâmetros forças de tração média e máxima. SILVA et al. (2000) encontraram na maior velocidade de semeadura do milho (quatro linhas), os maiores valores de força de tração.

Observa-se que, em cada estado de compactação, os valores de força de tração por linha das culturas de soja e milho diferem entre si, devido, principalmente, à variação da profundidade e da velocidade de deslocamento. Entretanto, para a demanda de potência por linha, essa diferença ocorre somente para o tratamento $\mathrm{T} 1$; isso pode ter ocorrido devido aos maiores valores de velocidade média encontrados nesse tratamento, que obteve acréscimo de velocidade de 3,36 e $3,72 \%$ nas culturas de soja e de milho, respectivamente, em relação ao tratamento T0. Esses dados são semelhantes aos observados por CASÃO JÚNIOR et al. (1998), que apresentaram diferença significativa na força de tração requerida por uma semeadora com quatro linhas de milho e sete de soja a 4,5 $\mathrm{km} \mathrm{h}^{-1}$. BORTOLLOTO et al. (2005) apresentaram resultados semelhantes para aveia, na velocidade de $10,21 \mathrm{~km} \mathrm{~h}^{-1}$, e SILVEIRA et al. (2005) verificaram que, com o aumento da profundidade de deposição de 0,197 para $0,268 \mathrm{~m}$, o requerimento de força de tração cresceu de 3,78 para $5,51 \mathrm{kN}$.

MERCANTE et al. (2005) verificaram que a velocidade de deslocamento quando passa de 5,20 para $8,45 \mathrm{~km} \mathrm{~h}^{-1}$, somente a potência na barra aumenta significativamente. MAHL et al. (2004) revelaram que, quando a velocidade passa de 4,4 para $8,1 \mathrm{~km} \mathrm{~h}^{-1}$, há incremento na potência na barra, de $96 \%$.

O valor máximo de força de tração por linha, para as duas culturas, pode ter ocorrido devido às irregularidades e às desuniformidades do terrreno, aos embuchamentos momentâneos ou mesmo às diferenças na resistência do solo ao corte (LEVIEN, 1999). Nota-se, também, que, com o aumento do número de linhas utilizadas na semeadura da soja e do milho, há acréscimo da força de tração, utilizando o mesmo mecanismo sulcador. Esses dados estão em acordo com estudos realizados por MODOLO et al. (2005), que verificaram que há aumento do esforço na barra de tração com o acréscimo do número de linhas de semeadura, utilizando o mesmo mecanismo sulcador.

$\mathrm{Na}$ Tabela 5, são apresentados os resultados da população inicial aos 18 D.A.S. para as culturas de soja e de milho. Observa-se que os tratamentos em estudo não interferiram na população inicial da cultura da soja, porém, na cultura do milho, nota-se que a população inicial foi alterada significativamente nos tratamentos T3 e T5, quando relacionados com o tratamento T0. Mesmo não sendo verificada diferença significativa entre os tratamentos $\mathrm{T} 0$ e $\mathrm{T} 1$, esses possuem os maiores valores de população inicial, cerca de 3,29 e 2,03\% em relação ao tratamento T0 da cultura da soja; no entanto, para a cultura do milho, nota-se comportamento diferenciado para esses tratamentos, pois a população do milho é reduzida cerca de 12,91 e $25,08 \%$. Apesar de a cultura de soja obter maiores valores de demanda energética, esses não interferiram na população inicial, enquanto, no milho, ocorreu interferência principalmente no tratamento T3 e T5.

TABELA 5. População inicial de plantas $\left(\mathrm{pl} \mathrm{ha}^{-1}\right)$ de soja e milho nos quatro estados de compactação. Initial population plant $\left(\mathrm{pl} \mathrm{ha}^{-1}\right)$ of soybeans and corn in the four compaction states.

\begin{tabular}{ccl}
\hline Tratamentos & Soja & Milho \\
\hline T0 & $365.333 \mathrm{a}^{*}$ & $86.444 \mathrm{a}$ \\
T1 & $377.777 \mathrm{a}$ & $76.555 \mathrm{ab}$ \\
T3 & $372.888 \mathrm{a}$ & $69.111 \mathrm{~b}$ \\
T5 & $362.888 \mathrm{a}$ & $72.111 \mathrm{~b}$ \\
C.V. $(\%)$ & 16,36 & 19,56 \\
\hline
\end{tabular}

*Médias de tratamentos seguidas de mesma letra na coluna não diferem entre si, pelo teste $t$-Student $(\mathrm{P}>0,05)$. 
De acordo com os resultados expostos anteriormente, verifica-se que os valores máximos de resistência do solo à penetração $(2,50$ e 3,00 MPa), na profundidade 0,07 a $0,12 \mathrm{~m}$, e densidade do solo máxima de $1,39 \mathrm{Mg} \mathrm{m}^{-3}$, na profundidade de 0,10 a $0,20 \mathrm{~m}$, não interferiram no estabelecimento da população inicial de plantas de soja; no entanto, para o milho, ocorreu redução da população inicial nos tratamentos T3 e T5, e ainda os maiores valores de força de tração e potência na barra também não influenciaram na população inicial da soja.

MAHL et al. (2004) relataram resultados diferentes para o estande inicial do milho em estudo de diferentes sistemas de preparo do solo e de velocidade, em que verificaram que os tratamentos não interferiram na população inicial do milho. KLEIN et al. (2002) concluíram que a velocidade de semeadura de soja não afetou significativamente o número de plântulas emergidas aos 27 dias após a semeadura.

\section{CONCLUSÕES}

Os valores de resistência do solo à penetração de 2,50 e 3,00 $\mathrm{MPa}$ e densidade do solo máxima de $1,39 \mathrm{Mg} \mathrm{m}^{-3}$ não interferiram no estabelecimento da população inicial de plantas de soja; no entanto, para o milho, ocorreu redução nos tratamentos.

À medida que ocorreu variação na profundidade de sulcamento e na velocidade de deslocamento, ocorreu aumento da força de tração por linha para as duas culturas estudadas.

\section{AGRADECIMENTOS}

À Universidade Estadual do Oeste do Paraná (UNIOESTE) e à Prefeitura Municipal de Cascavel - PR.

\section{REFERÊNCIAS}

ASAE. AMERICAM SOCIETY OF AGRICULTURAL ENGINEERS. Agricultural machinery management, 1999. (ASAE S313.3)

BORTOLOTTO, V.C.; PINHEIRO NETO, R.; BORTOLOTTO, M.C. Demanda energética de uma semeadora-adubadora sob diferentes velocidades de deslocamento e tipos de cobertura vegetal no plantio da soja. Acta Scientiarum Agronomic, Maringá, v.27, n.2, p.357-62, 2005.

CASÃO JÚNIOR, R.; ARAÚJO, A.G.; RALISCH, R.; SILVA, A.L.; LADEIRA, A.S.; SILVA, J.C.; MACHADO, P.; ROSSETTO, R. Avaliação do desempenho da semeadora-adubadora Magnum 2850 PD no basalto paranaense. Londrina: IAPAR, 1998. (Circular, 105)

CEPIK, C.T.C.; TREIN, C.R.; LEVIEN, R. Força de tração e volume de solo mobilizado por haste sulcadora em semeadura direta sobre campo nativo, em função do teor de água no solo, profundidade e velocidade de operação. Engenharia Agrícola, Jaboticabal, v.25, n.2, p.447-57, 2005.

EMBRAPA. EMPRESA BRASILEIRA DE PESQUISA AGROPECUÁRIA. Manual e métodos de análise do solo. 2. ed. Rio de Janeiro, 1999b. 212 p.

EMPRESA BRASILEIRA DE PESQUISA AGROPECUÁRIA-EMBRAPA. Sistema brasileiro de classificação de solos. Brasília: EMBRAPA, 1999a. 20 p.

FURLANI, C.E.A.; LOPES, A.; SILVA, R.P da. Avaliação de semeadora-adubadora de precisão trabalhando em três sistemas de preparo do solo. Engenharia Agrícola, Jaboticabal, v.25, n.2, p. 458-64, 2005.

GENRO JÚNIOR, S.A.; REINERT, D.J.; REICHERT, J.M. Variabilidade temporal da resistência a penetração de um Latossolo Argiloso sob semeadura direta com rotação de culturas. Revista Brasileira de Ciência do Solo, Viçosa, v.28, n.2, p.487-84, 2004.

KLEIN, V.A.; SIOTA, T.A.; ANESE, A.L.; BARBOSA, R. Efeito da velocidade na semeadura direta da soja. Engenharia Agrícola, Jaboticabal, v.22, n.1, p.75-82, 2002. 
LEVIEN, R. Condições de cobertura e métodos de preparo do solo para a implantação da cultura do milho (Zea mays L.). 1999. 305 f. Tese (Doutorado em Energia na Agricultura) - Faculdade de Ciências Agronômicas, Universidade Estadual Paulista Júlio de Mesquita Filho, Botucatu, 1999.

MAHL, D.; GAMERO, C.A.; BENEZ, S.H.; FURLANI, C.E.A.; SILVA, A.R.B. Demanda energética e eficiência da distribuição de sementes de milho sob variação de velocidade e condição de solo. Engenharia Agrícola, Jaboticabal, v.24, n.1, p.150-7, 2004.

MERCANTE, E.; SILVA, S. de L.; MODOLO., A.J.; SILVEIRA, J.C.M. Demanda energética e distribuição de sementes de milho em função da velocidade de duas semeadoras. Revista Brasileira de Engenharia Agrícola Ambiental, Campina Grande, v.9, n.3, p.424-8, 2005.

MODOLO, A.J.; GABRIEL FILHO, A.; SILVA, S. de L.; GNOATTO, E. Força de tração necessária em função do número de linhas de semeadura utilizadas por uma semeadora-adubadora de precisão. Engenharia Agrícola, Jaboticabal, v.25, n.2, p.465-73, 2005.

OLIVEIRA, M.F.B.; SIQUEIRA, R.; RALISCH, R.; ARAÚJO, A.G.; CASÃO JÚNIOR, R. Mobilização do solo por hastes sulcadoras de semeadoras-adubadoras de plantio direto. In: CONGRESSO BRASILEIRO DE ENGENHARIA AGRÍCOLA, 29., 2000, Fortaleza. Anais... Fortaleza: Sociedade Brasileira de Engenharia agrícola, 2000. 1 CD-ROM.

REINERT, D.J.; REICHERT, J.M.; SILVA, V.R. Propriedades fisicas de solos em sistema plantio direto irrigado. Santa Maria: Palloti, 2001. v.1, p.114-33.

SECCO, D.; REINERT, D.J.; REICHERT, J.M.; DA ROS, C.O. Produtividade de soja e propriedades físicas de um Latossolo submetido a sistemas de manejo e compactação. Revista Brasileira de Ciência do Solo, Viçosa, v.28, n.5, p.797-804, 2004.

SILVA, V.R. da. Propriedades físicas e hídricas em solos sob diferentes estados de compactação. 2003. 171 f. Tese (Doutorado em Biodinâmica e Manejo do Solo) - Universidade Federal de Santa Maria, Santa Maria, 2003.

SILVA, V.R.; REINERT, D.J.; REICHERT, J.M. Densidade do solo, atributos químicos e sistema radicular do milho afetado pelo pastejo e manejo do solo. Revista Brasileira de Ciência do Solo, Viçosa, v.24, n.1, p.191-9, 2000.

SILVA, R.H. da; ROSOLEN, C.A. Crescimento radicular de soja em razão da sucessão de cultivos e da compactação do solo. Pesquisa Agropecuária Brasileira, Brasília, v.37, n.6, p.855-60, 2002.

SILVEIRA, J.C.M. da; MONDOLO, A.J.; SILVA. S. de L.; GABRIEL FILHO, A. Força de tração e potência de uma semeadora em duas velocidades de deslocamento e duas profundidades de deposição. Revista Engenharia Agrícola e Ambiental, Campina Grande, v.9, n. 1, p.125-8, 2005.

SIQUEIRA, R.; ARAÚJO, A.G.; CASÃO JÚNIOR, R.; RALISCH, R. Desempenho energético de semeadoras-adubadoras de plantio direto na implantação da cultura da soja (Glycine max L.). In: CONGRESSO BRASILEIRO DE ENGENHARIA AGRÍCOLA, 30., 2001, Foz do Iguaçu. Anais... Foz do Iguaçu: Sociedade Brasileira de Engenharia Agrícola, 2001. 1 CD-ROM.

SOANE, B.D.; OUWERKERK, C. Soil compaction in crop production. Amsterdam: Elsevier, 1994. $662 \mathrm{p}$.

TIEPPO, R.C. Desenvolvimento de um penetrômetro manual eletrônico. 2004. 33 f. Dissertação (Mestrado em Engenharia de Sistemas Agroindustriais) - Universidade Estadual do Oeste do Paraná, Cascavel, 2004.

TRINTIN, C.G.; NETO PINHEIRO, R.; BORTOLOTTO, V.C. Demanda energética solicitada por uma semeadora - adubadora para plantio direto, submetida a três velocidades de operação. Acta Scientiarum Agronomy, Maringá, v.27, n.1, p.127-31, 2005. 